\title{
Bioinformatics identification of the candidate microRNAs and construction of a competing endogenous RNA regulatory network in lacrimal gland adenoid cystic carcinoma high-grade transformation
}

\author{
MEIXIA JIANG ${ }^{1 *}$, XUN LIU $^{1 *}$, CHUANLI ZHANG ${ }^{1}$, LIMIN ZHU $^{1}$, HAI-DONG WU ${ }^{2}$, \\ LIJIE DONG $^{1}$, TINGTING WANG ${ }^{1}$, TINGTING LIN ${ }^{1}$ and YANJIN HE ${ }^{1}$ \\ ${ }^{1}$ Tianjin International Joint Research and Development Centre of Ophthalmology and Vision Science, \\ Eye Institute and School of Optometry, Tianjin Medical University Eye Hospital; \\ ${ }^{2}$ Tianjin Key Laboratory of Early Draggability Evaluation of Innovative Drugs, \\ Tianjin International Joint Academy of Biomedicine, Tianjin 300384, P.R. China
}

Received July 29, 2020; Accepted December 9, 2020

DOI: $10.3892 / 01.2021 .12621$

\begin{abstract}
Adenoid cystic carcinoma of the lacrimal gland (LACC) is a major orbital malignancy. The recurrence rate and mortality rate are higher in LACC high-grade transformation (LACC-HGT) compared with in LACC. The present study aimed to identify the candidate microRNAs (miRNAs/ miRs) and construct a competing endogenous RNA (ceRNA) regulatory network for LACC-HGT. A miRNA microarray on paraffin-embedded tissues was performed to identify the differentially expressed miRNAs (DEMs) of LACC-HGT. The overlap with the salivary adenoid cystic carcinoma miRNA/ RNA sequencing dataset in the Gene Expression Omnibus was used to identify candidate miRNAs. In order to construct a ceRNA regulatory network of LACC-HGT, a microarray of mRNA and circRNA in primary cell lines was performed. The circRNAs and genes with high expression in LACC-HGT were predicted as targeting miRNAs, and the circRNA-miRNAmRNA regulatory network was constructed. miR-140-3p was identified as part of the ceRNA network and as a candidate miRNA, therefore this was further analyzed using reverse transcription-quantitative (RT-q)PCR. Overall, the Agilent
\end{abstract}

Correspondence to: Dr Tingting Lin or Dr Yanjin He, Tianjin International Joint Research and Development Centre of Ophthalmology and Vision Science, Eye Institute and School of Optometry, Tianjin Medical University Eye Hospital, 251 Fu Kang Road, Tianjin 300384, P.R. China

E-mail: 1tt6123@126.com

E-mail: yhe04@tmu.edu.cn

*Contributed equally

Key words: lacrimal gland adenoid cystic carcinoma with high-grade transformation, microarray, competing endogenous RNA
Human microarray analysis identified a total of 16 DEMs from the LACC-HGT paraffin-embedded tissues. A total of 653 DECs and 9,566 DEGs of LACC-HGT primary cell lines were screened via the microarray of mRNA and circRNA. The ceRNA regulatory network was constructed using the cross-binding of circRNA-miRNA, miRNA-mRNA and the downregulated miRNAs in LACC-HGT to clearly demonstrate the circRNA-miRNA-mRNA interaction relationship. RT-qPCR results confirmed that miR-140-3p was downregulated in LACC-HGT tissues and primary cell lines compared with LACC. Target genes CD200 and parathyroid hormonerelated protein were significantly upregulated in LACC-HGT primary cell lines. miR-140-3p and its target genes may play an important role in LACC-HGT pathogenesis. In conclusion, the current bioinformatics study constructed a ceRNA network based on a microarray, which may help identify novel miRNA therapeutic targets for LACC-HGT.

\section{Introduction}

Lacrimal gland adenoid cystic carcinoma (LACC) is the most common form of malignant epithelial lacrimal gland tumor. It is characterized by a slow progression, local invasion and metastasis, and usually has a poor prognosis due to osseous and perineural invasion $(1,2)$. A study in the University of Texas MD Anderson Cancer Center shows the local recurrence rate is $35 \%$, the distant metastasis rate is $80 \%$ and the mortality is $65 \%(3,4)$. Local recurrence and distant metastatic disease occur in $~ 50 \%$ of patients within 5 to 10 years, and currently there is no effective treatment $(5,6)$. Despite classic histological types, ACC can also show areas of classic ACC with a gradual or sharp transition to the areas of high-grade carcinoma. Initially, this atypical ACC was described as 'hybrid tumors', subsequently as 'dedifferentiated' and most recently as high-grade transformation (HGT) (7-9). The histological features of ACC with HGT are composed of two histological components: Conventional low-grade ACC 
and high-grade 'dedifferentiated' tumor tissues (10). LACC rarely undergoes HGT, but there is evidence that histological subtypes are associated with prognosis. A study shows the lymph node metastasis rate of LACC-HGT is higher compared with LACC (43-57\% vs. 5-25\%) (11). According to a previous report, LACC-HGT accelerates the process of tumor local recurrence and distant metastasis, resulting in increased mortality, shortened postoperative survival and poor outcomes of conventional treatment (12).

Non-coding RNAs (ncRNAs) are generally defined as a wide class of RNAs without protein-coding function, but these have regulatory functions in the various biological processes, such as post-transcriptional regulators, regulation of mitochondrial biology and metabolic control. As a group of endogenous, single-stranded, small non-coding RNA molecules, microRNAs (miRNAs/miRs) negatively regulate gene expression at the post-transcriptional level (13). Previous studies have shown significant differences in miRNA expression of ACC. For example, miR-24-3p was low expression in high metastasis type ACC cells, and further research reported that miR-24-3p is downregulated in LACC tissue and inhibits ACC cell proliferation, migration and invasion through targeting protein kinase C eta type (14). Meanwhile, downregulated miR-24-3p also inhibits the epithelial-mesenchymal transition (EMT) process of ACC. Most recently, Hao et al (15) demonstrated that miR-93-5p is overexpressed in tissues and plasma of patients with LACC compared with healthy controls. miR-93-5p promotes LACC cell migration, invasion and proliferation by targeting the downregulation of breast cancer metastasis-suppressor 1-like protein through regulation of the Wnt signaling pathway. Circular RNAs (circRNAs) are novel non-coding RNAs that have been reported to be involved in the progression of various cancers, such as non-small cell lung carcinoma, bladder cancer and osteosarcoma (16). However, the regulatory mechanisms of circRNAs in LACC remain unclear.

The hypothesis of ceRNA was defined by Salmena et al (17) in 2011. ceRNA introduced a new crosstalk theory of gene regulation that promotes the physiology and development of diseases, such as cancer. Recent studies about ceRNA have expanded our insights into LACC-HGT pathogenesis (18).

\section{Materials and methods}

Patients and tissue samples. A total of patients with 12 LACC were originally included in the study, all patients benefited from a surgical resection. HGT occurred in six cases following the histological analysis. All patient profiles were evaluated; their symptoms and signs were assessed as two separate components, and two patients were lost during follow-up in the LACC group. A total of six LACC paraffin-embedded tissue samples and six LACC-HGT paraffin-embedded tissue samples were provided by Tianjin Medical University Eye Hospital (Tianjin, China) from June 2009 to May 2017. Informed written consent for research purposes was obtained from the patients before tissue collection. The Institutional of Human Research Ethics Committee of the Tianjin Medical University Eye Hospital approved the study [Tianjin, China; approval no. 2017KY(L)-23].

Primary cell line culture. Primary cell line culture was performed as described previously (19) via tissue block culture techniques, and the cells were cultured in RPMI-1640 medium with $10 \%$ fetal bovine serum and $1 \%$ penicillin/streptomycin solution (both HyClone; Cyvita) at $37^{\circ} \mathrm{C}$ in a $5 \% \mathrm{CO}_{2}$ incubator. Only LACC and LACC-HGT primary cells in passages 4-10 were used for further experiments.

miRNA microarray analysis. Differentially expressed miRNAs (DEMs) of 12 LACC samples were detected using the Agilent Human miRNA Microarray, Release 21.0 (8*60K, Design ID: 070156). miRNA expression profiles (GSE59700) of salivary adenoid cystic carcinoma (SACC) were obtained from the GEO (http://www.ncbi.nlm.nih.gov/geo) (20). The dataset contains 12 SACC samples with matched normal tissues.

Candidate miRNAs identification. In order to get raw data of miRNA microarray, the array images were imported to Feature Extraction software (version 10.7.1.1, Agilent Technologies). Genespring software (version 13.1; Agilent Technologies) was employed to finish the basic analysis with the raw data. First, the raw data was normalized with the quantile algorithm. DEMs were then identified through the ratio of the expression quantity (fold-change; FC) as well as P-values calculated using unpaired t-tests, these data are presented in Table IV. The threshold set for up and downregulated DEMs was a $\log 2 \mathrm{FCl}>1$ and a $\mathrm{P} \leq 0.05$. GEO2R is one of the powerful tools to screen the DEMs (21). Datasets of SACC downloaded from the GEO database were analyzed by GEO2R. Candidate miRNAs were selected from the overlap between the miRNA microarray and the DEMs from the GEO datasets.

LACC-HGT differentially expressed circRNAs (DECs) and genes screening. The DECs and differentially expressed genes (DEGs) of LACC-HGT primary cell lines were detected using the Agilent Human Microarray 2018 (4*180k, Design ID: 085630). To begin with, the raw data was normalized with the quantile algorithm. DECs and DEGs were then identified through fold-change as well as P-values calculated using unpaired t-tests, these data are presented in Tables SII and SIII. Upregulated and downregulated circRNAs and genes were those with an absolute value of logarithmic transformed fold-change (FC) $[\log 2 \mathrm{FC} \geq \log 2(1.5)]$ and $\mathrm{P} \leq 0.05$.

Functional enrichment analysis. Gene Ontology (GO) and Kyoto Encyclopedia of Genes and Genomes (KEGG) analyses were applied to determine the roles of these DECs and DEGs. Afterwards, hierarchical clustering was performed to display the distinguishable genes' expression pattern among samples. The hypergeometric distribution algorithm was used to calculate the significance of different gene enrichment in each GO term and each pathway (22). The result of this calculation gave P-values of enriched significance, and the lower the value the more statistically significant it is.

Gene Set Enrichment Analysis (GSEA). The expression profile of genes provided the ratio of the expression quantity $(\log 2 \mathrm{FC})$ and then the genes were arranged in descending order. The GSEA algorithm was used to identify the more variable pathways and gene set of LACC-HGT compared with LACC. 
Interaction prediction of circRNA-miRNA and miRNA-mRNA. To improve the predictive accuracy, CircInteractome (23) and ENCORI (http://starbase.sysu.edu.cn/) were used to predict the circRNA-miRNA targeting. The miRWalk (http://mirwalk.umm.uni-heidelberg.de/) database was used to predict miRNA-mRNA targeting. In this analysis, as the DEGs identified totaled $>5,000$, exceeding the limit of this tool, the top 500 genes were selected for prediction.

Construction of ceRNA regulatory network. According to the default screening criteria $\mid \log 2 \mathrm{FCl}>1$ and $\mathrm{P}<0.05$, only five downregulated miRNAs were obtained, which did not meet the subsequent analysis requirements. Therefore, the screening standard was adjusted to $\log 2 \mathrm{FCl}>1$ and $\mathrm{P}<0.2$, and a total of 31 miRNAs with significant downregulation were screened for ceRNA regulatory network construction. The previously obtained circRNA-miRNA, miRNA-mRNA and downregulated miRNAs in LACC-HGT were imported into Cytoscape 3.7.2 software (24) to map the ceRNA regulatory network. A Sankey map was drawn.

Protein-protein interaction (PPI) network of proteins in ceRNA regulatory network. The proteins in the ceRNA regulatory network were imported into STRING (25) for PPI network construction, and the interaction network was plotted using Cytoscape 3.7.2 software.

RNA extraction and reverse transcription-quantitative (RT-q) $P C R$. As a candidate miRNA, miR-140-3p was also involved in ceRNA regulatory network, therefore the expression of miR-140-3p in LACC-HGT tissues and primary cells was analyzed. miRNAs of tissues and primary cells were extracted using the miRcute miRNA Isolation kit (Tiangen Biotech Co., Ltd.). The RNA sample was reversely transcribed into the first-strand cDNA using the miRcute Plus miRNA First-Strand cDNA kit (Tiangen Biotech Co., Ltd.). The temperature protocol was follows: $42^{\circ} \mathrm{C}$ For $3 \mathrm{~min}, 42^{\circ} \mathrm{C}$ for $15 \mathrm{~min}$ and $95^{\circ} \mathrm{C}$ for 3 min. Quantification analysis was performed using miRNA SYBR Green PCR master mix (Tiangen Biotech Co., Ltd.), the temperature protocol was follows: $95^{\circ} \mathrm{C}$ For $15 \mathrm{~min}, 95^{\circ} \mathrm{C}$ for $10 \mathrm{sec}), 60^{\circ} \mathrm{C}$ for $30 \mathrm{sec}$ for 40 cycles. Total RNA of LACC and LACC-HGT primary cells were extracted using the EZ-press RNA Purification kit (EZBioscience). The RNA sample was reversely transcribed into the first-strand cDNA using the FastQuant RT kit (Tiangen Biotech Co., Ltd.). Quantification analysis was performed using SYBR Green PCR master mix (Tiangen Biotech Co., Ltd.). and specific primers on Roche LightCycler ${ }^{\circledR} 96$ Sequence Detection system (Roche Diagnostics). The forward primers for miR-140-3p was 5'-GCCCGTGGTTCT ACCCT-3'; for 5 sec was 5'-GGAGACCGCCTGGGAATA-3'. The reverse primers for these were universal primers included in the kit. Primers are shown in Table I. Expression levels were quantified using the $2^{-\Delta \Delta \mathrm{Cq}}$ method with and GAPDH as internal control (26), these data are presented in the Fig. 6.

Statistical analysis. Each experiment was performed at least three times, the data are presented as mean $\pm \mathrm{SD}$, and statistical analyses were performed using SPSS version 21 (IBM Corp) and GraphPad Prism version 7.00 (GraphPad Software). The analysis of clinical and pathological dates was determined
Table I. Primer sequences.

\begin{tabular}{ll}
\hline Primers & \multicolumn{1}{c}{ Sequence, $5^{\prime} \rightarrow 3^{\prime}$} \\
\hline miR-140-3p & GCCCGTGGTTCTACCCT \\
$5 \mathrm{~s}$ & GGAGACCGCCTGGGAATA \\
CD200 & \\
Forward & AAGTGGTGACCCAGGATGAAA \\
Reverse & AGGTGATGGTTGAGTTTTGGAG \\
PTHLH & \\
Forward & ATTTACGGCGACGATTCTTCC \\
Reverse & GCTTGGAGTTAGGGGACACC \\
GAPDH & \\
Forward & GATGCTGGCGCTGAGTACG \\
Reverse & GCTAAGCAGTTGGTGGTGC \\
\hline
\end{tabular}

PTHLH, parathyroid hormone-related protein.

using Fisher's exact tests. Independent samples unpaired t-tests were used to screen the DECs, DEMs and DEGs. The results of RT-qPCR were also analyzed using unpaired Student's t-test (Fig. 6). $\mathrm{P}<0.05$ was considered to indicate a statistically significant difference.

\section{Results}

Patient characteristics. In this study, 12 patients clinically diagnosed with LACC were enrolled. These included six cases of LACC and six cases of LACC-HGT. There were differences between LACC group and LACC-HGT group. Briefly, the mean age of patients with LACC was 55.5 years, with the range between 29 and 68 years, and the mean age of the patient with LACC-HGT was 38.5 years, with a range between 23 and 50 years. There were three cases in the right eye and three cases in the left eye in LACC group and the same for the LACC-HGT group. Main symptoms of LACC included pain or dysesthesia, ocular proptosis, palpation, eye movement disorder and blurred vision (Table II). The pathological grade was higher in the LACC-HGT (all were grade III) compared with the LACC group and the difference was statistically significant $(\mathrm{P}<0.05$; Table III). IHC showed decreased expression of p63 and smooth muscle actin in LACC-HGT, and an increased number of Ki-67 positive cells in LACC-HGT group, but this was not significant ( $\mathrm{P}>0.05)$. The mortality of LACC-HGT group was higher compared with that of LACC group, the mortality of LACC was $50 \%$ and the mortality of LACC-HGT was $83 \%$. A summary of clinical and pathological features of the 12 patients is listed in Tables II and III. The results indicated that the patients with HGT had a more aggressive disease compared with conventional LACC, accompanied by a poorer prognosis. The recurrent cases in 5 years and the death rate of the LACC-HGT group $(3,83 \%)$ were higher compared with the LACC group $(2,50 \%)$. However, the difference was not statistically significant.

Differentially expressed miRNAs. The results showed that there were differences in miRNA expression between LACC and LACC-HGT groups. A total of 16 DEMs in LACC-HGT 
Table II. Clinical features of the 12 patients.

\begin{tabular}{|c|c|c|c|}
\hline Clinical features & LACC-HGT & LACC & P-value \\
\hline Total number of patients & 6 & 6 & \\
\hline Sex & & & 0.83 \\
\hline Male & 2 & 3 & \\
\hline Female & 4 & 3 & \\
\hline Median age, years & 38.5 & 55.5 & 0.07 \\
\hline Tumor side & & & 0.83 \\
\hline Left & 3 & 2 & \\
\hline Right & 3 & 4 & \\
\hline Duration of symptoms, mean months & 5.0 & 10.5 & \\
\hline Symptom & & & 0.73 \\
\hline Pain or dysesthesia & 4 & 4 & \\
\hline \multicolumn{4}{|l|}{ Signs } \\
\hline Ocular proptosis, mean mm & 3.67 & 3.17 & \\
\hline Palpation & 5 & 5 & 0.78 \\
\hline Eye movement disorder & 4 & 5 & 0.50 \\
\hline Blurred vision & 3 & 4 & 0.50 \\
\hline \multicolumn{4}{|l|}{$\mathrm{CT}$} \\
\hline Periosteum involvement & 5 & 4 & 0.50 \\
\hline Bony involvement & 3 & 4 & 0.50 \\
\hline \multicolumn{4}{|l|}{ MRI } \\
\hline Brain & 1 & 2 & 0.50 \\
\hline Temporal fossa & 2 & 0 & 0.23 \\
\hline Sinus & 1 & 2 & 0.50 \\
\hline Ocular muscle & 3 & 5 & 0.28 \\
\hline Follow up time, months & 40.2 & $70.8^{\mathrm{a}}$ & \\
\hline Cases of death & 5 & 2 & 0.33 \\
\hline Death rate, $\%$ & 83 & $50^{\mathrm{a}}$ & 0.67 \\
\hline Cases of recurrence in 5 years & 3 & 2 & 0.74 \\
\hline Recurrence rate of 5 years, $\%$ & 50 & $50^{\mathrm{a}}$ & 0.83 \\
\hline \multicolumn{4}{|l|}{ Survival time, years } \\
\hline 2 & 3 & $3^{\mathrm{a}}$ & 0.45 \\
\hline 5 & 1 & $3^{\mathrm{a}}$ & 0.23 \\
\hline
\end{tabular}

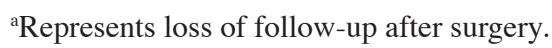

paraffin-embedded tissues were screened by microarray, including five downregulated miRNAs and 11 upregulated miRNAs, their expressions are shown in Table IV. Overall, 704 DEMs of SACC tissues were screened from GEO database (Table SI).

Candidate miRNAs of LACC-HGT. A total of four DEMs that could be candidate miRNAs of LACC-HGT were selected from the overlap between the miRNA microarray and GEO dataset (Fig. 1A). They were hsa-miR-140-3p, -140-5p, -574-3p and -636. Among them, hsa-miR-140-3p, -140-5p and $-574-3 p$ were downregulated in the LACC-HGT group and hsa-miR-636 was upregulated in LACC-HGT group.

DECs and DEGs. The expression of circRNAs and genes in LACC and LACC-HGT primary cell lines were significantly different. After being compared with LACC primary cell lines, the results of circRNA microarray showed that there were 258 upregulated circRNAs, 395 downregulated circRNAs (Fig. 1B; Table SII, and 5,162 upregulated and 4,404 downregulated genes (Fig. 1C) in LACC-HGT primary cell lines (Table SIII).

GO and pathway analysis of DECs and DEGs. GO and KEGG pathway analyses were performed in DECs and DEGs, respectively. The results indicated that the DECs are mainly located in 'cytosol', 'membrane' and 'nucleoplasm', the upregulated circRNAs are closely associated with 'translational initiation', 'RNA binding', 'regulation of $\mathrm{G}_{2} / \mathrm{M}$ transition' and 'mitotic spindle assembly' (Fig. 2A), and mainly involved in 'RNA transport', 'RNA degradation' and 'cell cycle' processes (Fig. 2B). The downregulated circRNAs were associated with 
Table III. Pathological features of the 12 patients.

\begin{tabular}{lccc}
\hline Clinical features & LACC-HGT & LACC & P-value \\
\hline Histological grading & & & $<0.01$ \\
I & 0 & 4 & \\
II & 0 & 2 & \\
III & 6 & 0 & \\
TNM & & & 0.72 \\
T2N0M0 & 1 & 2 & \\
T4aN0M0 & 2 & 1 & \\
T4bN0M0 & 1 & 2 & \\
T4cN0M0 & 2 & 1 & \\
IHC, \% & & & \\
SMA & 14.3 & 45 & $<0.01$ \\
p63 & 16.8 & 53.3 & $<0.01$ \\
p53 & 17 & 21 & 0.74 \\
Ki-67 & 26.7 & 11.8 & 0.05 \\
\hline
\end{tabular}

TNM, Tumor-Node-Metastasis; LACC, lacrimal gland adenoid cystic carcinoma; HGT, high-grade transformation; SMA, smooth muscle actin.

Table IV. Expressions of differentially expressed miRs.

\begin{tabular}{llrrr}
\hline ID & Regulation & FC & Log FC & P-value \\
\hline hsa-miR-140-3p & Down & -8.08 & -3.01 & 0.02 \\
hsa-miR-140-5p & Down & -6.35 & -2.67 & 0.04 \\
hsa-miR-3653-3p & Down & -4.04 & -2.01 & 0.07 \\
hsa-miR-574-3p & Down & -2.20 & -1.14 & 0.03 \\
hsa-miR-29a-3p & Down & -2.09 & -1.07 & 0.01 \\
hsa-miR-6785-5p & Up & 2.03 & 1.02 & 0.05 \\
hsa-miR-4651 & $\mathrm{Up}$ & 1.64 & 0.71 & 0.04 \\
hsa-miR-4459 & $\mathrm{Up}$ & 2.23 & 1.15 & 0.08 \\
hsa-miR-7114-3p & $\mathrm{Up}$ & 3.50 & 1.81 & 0.04 \\
hsa-miR-636 & $\mathrm{Up}$ & 3.67 & 1.87 & 0.04 \\
hsa-miR-6779-5p & $\mathrm{Up}$ & 5.35 & 2.42 & 0.02 \\
hsa-miR-135a-3p & $\mathrm{Up}$ & 4.51 & 2.17 & 0.03 \\
hsa-miR-1469 & $\mathrm{Up}$ & 4.18 & 2.06 & 0.02 \\
hsa-miR-4488 & $\mathrm{Up}$ & 9.26 & 3.21 & $<0.01$ \\
hsa-miR-4322 & $\mathrm{Up}$ & 8.00 & 3.00 & 0.02 \\
hsa-miR-6075 & $\mathrm{Up}$ & 4.51 & 2.17 & 0.02 \\
\hline
\end{tabular}

miR, microRNA; FC, fold-change; down, downregulated; up, upregulated.

'nuclear export signal receptor activity', 'focal adhesion' and 'protein export from nucleus' (Fig. 2C), and mainly involved in the 'Relaxin signaling pathway', 'EGFR tyrosine kinase inhibitor resistance' and 'B cell receptor signaling pathway' (Fig. 2D). DEGs were mainly located in 'plasma membrane', 'extracellular matrix', 'basement membrane and cytoplasm', the upregulated genes were associated with 'type I interferon signaling pathway', 'NF-кB transcription', 'extracellular matrix structural constituent' and 'cell proliferation' (Fig. 2E), and mainly involved in 'protein digestion and absorption', 'TNF signaling pathway', 'p53 signaling pathway' and 'MAPK signaling pathway' processes (Fig. 2F). The downregulated genes were associated with 'extracellular matrix organization', 'cell-cell adhesion' and 'EMT' (Fig. 2G), and mainly involved in 'pathways in cancer', 'focal adhesion' and 'Rap1 signaling pathway' (Fig. 2H).

GSEA of DEGs. GSEA of DEGs revealed that the 'interferon $\alpha$ response pathway,' 'response to extracellular matrix' and 'TNF signaling pathway' were significantly upregulated in the LACC-HGT group (Fig. 3A). However, the 'IL-3 pathway', 'focal adhesion', 'collagens' and 'signaling by PDGF' pathway were significantly downregulated (Fig. 3B). In addition, some metabolic pathways, including ' $\alpha$-Linolenic acid metabolism', 'ascorbate and alginate metabolism', 'fructose and mannose metabolism' and 'linoleic acid metabolism' were upregulated, as was the 'cytosolic DNA-sensing pathway' (Fig. 3C).

ceRNA regulatory network in $L A C C-H G T$. In order to construct ceRNA regulation networks, the screening criteria of DEMs were adjusted to $\mid \log 2 \mathrm{FCl}>1$ and $\mathrm{P}<0.2$. Ultimately, 31 miRNAs were downregulated in LACC-HGT group according to the microarray data (Fig. 4A). A total of eight circRNA-miRNA and 870 miRNA-mRNA interaction pairs were predicted from CircInteractome, ENCORI and miRWalk databases. circRNA-miRNA-mRNA ceRNA regulatory networks were constructed using the overlapping results between the present 31 downregulated miRNAs and the aforementioned circRNA-miRNA-mRNA interaction pairs, and 77 regulatory triplets (circRNA-miRNA-mRNA) were obtained (Fig. 4B). Finally, the ceRNA network that involved four circRNAs (hsa_circ_0079694,_0031983,_0050998 and _0010187), seven miRNAs (hsa-miR-140-3p, -199a-5p, -214-3p, $-221-3 p,-29 a-3 p,-34 c-5 p$ and $-423-5 p)$ and 235 genes was visualized using Cytoscape software. At the same time, in order to more clearly demonstrate the circRNA-miRNA-mRNA interaction, $\sim 100$ genes with the most significant upregulation were identified and a Sankey map was drawn (Fig. 4C).

PPI network of proteins in ceRNA regulatory network. PPI networks were established using the proteins involved in the ceRNA regulatory network, 126 nodes and 261 edges in total constituted the PPI network (Fig. 5). In a PPI network, each circle represents a protein, and edges represent interactions. The GO annotation of these proteins is marked in the figure to identify proteins with similar effects. Different colors in the circle represent different GO notes, and proteins of the same color have similar functions. Proteins with a higher 'connectivity degree' (interaction with other proteins) may be more important in the network. The interacting proteins of CD200 were identified such as TNF receptor associated factor 1 , receptor tyrosine kinase and hepatocyte growth factor.

Expression of miR-140-3p and its target genes. The data indicated that the expression of miR-140-3p was not significantly downregulated in LACC-HGT tissues ( $\mathrm{P}>0.05$; Fig. 6A), but was significantly downregulated in LACC-HGT primary cells $(\mathrm{P}<0.05$; Fig. 6B). miR-140-3p target genes CD200 and 

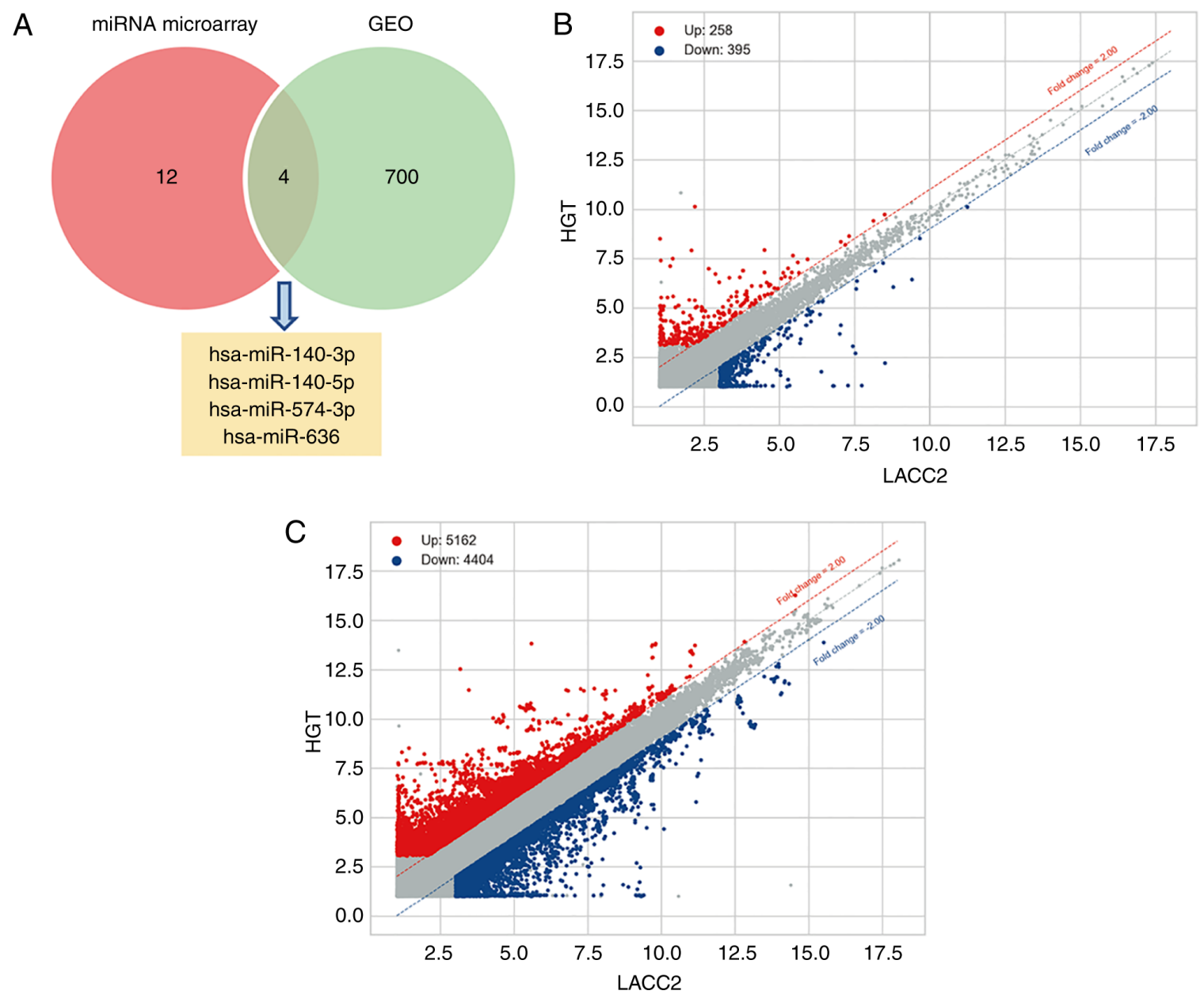

Figure 1. Candidate miRNAs, DECs and DEGs identification. (A) Venn diagram of candidate miRNAs selection between miRNA microarray and GEO dataset. (B) In total, 258 circRNAs were upregulated and 395 circRNAs were downregulated and (C) 5,162 genes were upregulated and 4,404 genes were downregulated in the lacrimal gland adenoid cystic carcinoma with high-grade transformation group. circRNA, circular RNA; DECs, differentially expressed circRNAs; DEGs, differentially expressed genes; GEO, Gene Expression Omnibus; miR, microRNA.

parathyroid hormone-related protein (PTHLH) were significantly upregulated $(\mathrm{P}<0.05$; Fig. 6C). GALNT12 has never been reported in lacrimal gland, and it was not detected in LACC and LACC-HGT primary cells.

\section{Discussion}

As effective diagnostic and treatment strategies have not been developed, the prognosis of patients with LACC remains poor, mostly due to local or distant metastasis and recurrence. Lacrimal gland carcinomas with HGT have been shown to be more aggressive compared with conventional carcinomas with a poorer prognosis, accompanied by higher local recurrence rate and propensity for cervical lymph node metastasis (27). However, the molecular genetic mechanisms responsible for the pathway of HGT in lacrimal gland carcinomas largely remain to be elucidated.

ncRNAs have an essential role in the function of oncogenes or tumor suppressor genes in cell signaling pathways. Previous studies have shown that ncRNAs can affect gene expression through RNA-RNA crosstalk-based ceRNA regulatory networks by competing for the miRNA response elements of miRNAs through an indirect post-transcriptional mechanism (28-30). miRNAs and circRNAs not only promote tumor progression, but it can also create the conditions that are not conducive to tumor development, thereby inhibiting tumor progression. This competitive mechanism theory is controversial, Han et al (31) reported that circMTO1 had no effect on the expression level of miR-9; however, an increasing number of studies have indicated that dysregulation of miRNAs is marker of cancer. Previous studies have revealed that miRNAs are commonly dysregulated in LACC, suggesting that these RNAs may play critical roles in tumorigenesis and the progression of LACC-HGT $(14,15)$. circRNAs can act as miRNA sponges to bind miRNAs and indirectly regulate mRNA expression (32). However, little is known about the role of circRNAs in LACC, and the present study is the first to report a ceRNA network of LACC-HGT, to the best of our knowledge. Therefore, comprehensively identifying the ncRNA profile and constructing ceRNA regulation networks based on the interactions among circRNAs, miRNAs and genes might provide novel insight into the pathogenesis of LACC-HGT.

The present study first analyzed the clinical and pathological data of 12 patients with LACC with or without HGT, and the results indicated that the patients with HGT had a more aggressive disease compared with conventional LACC, accompanied 
A

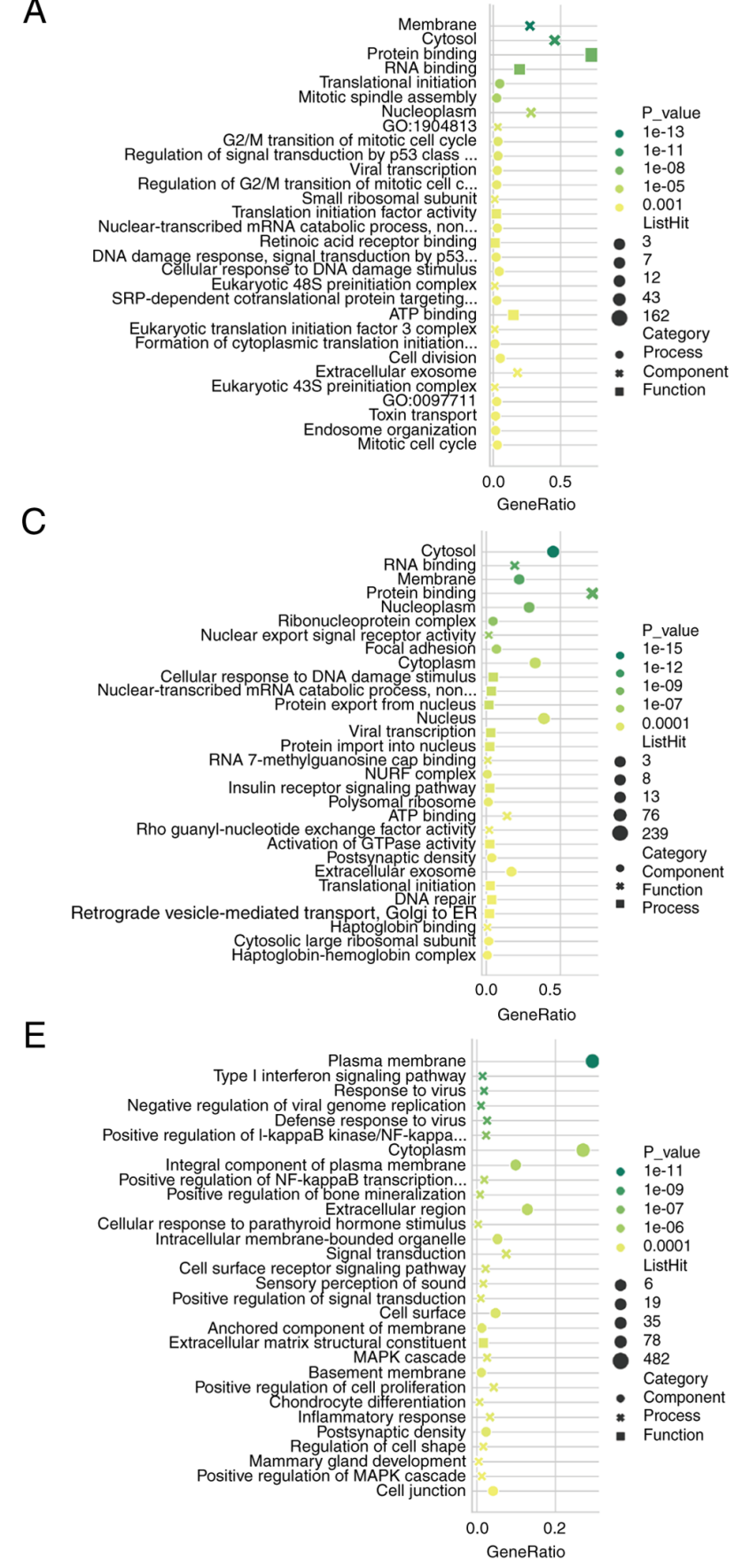

G

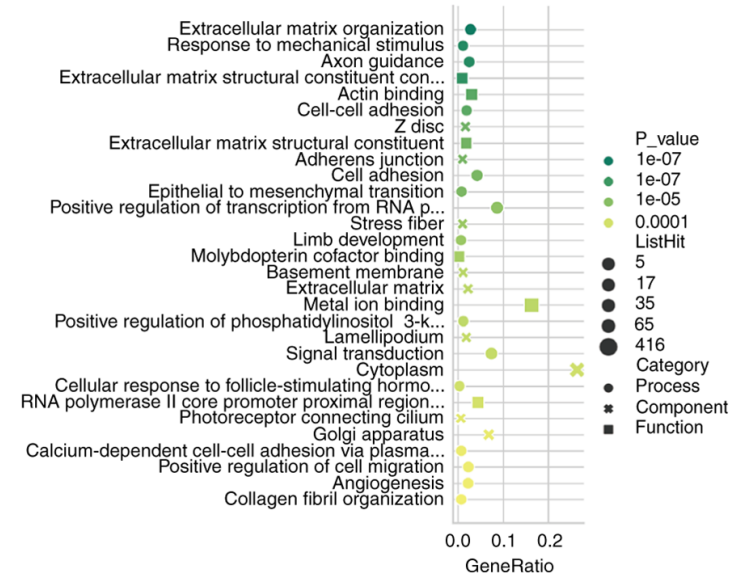

B

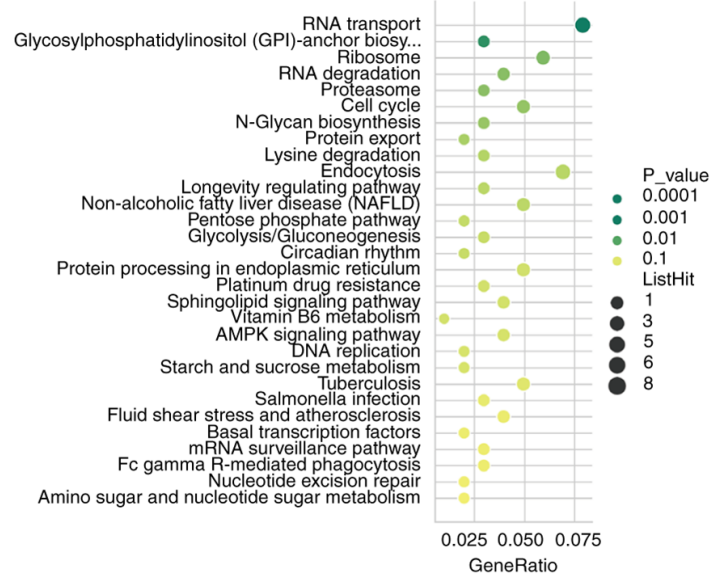

D

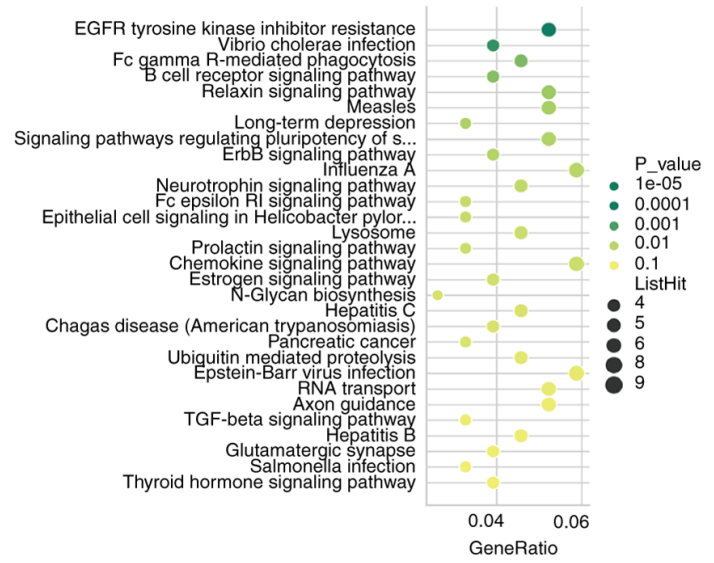

$\mathrm{F}$

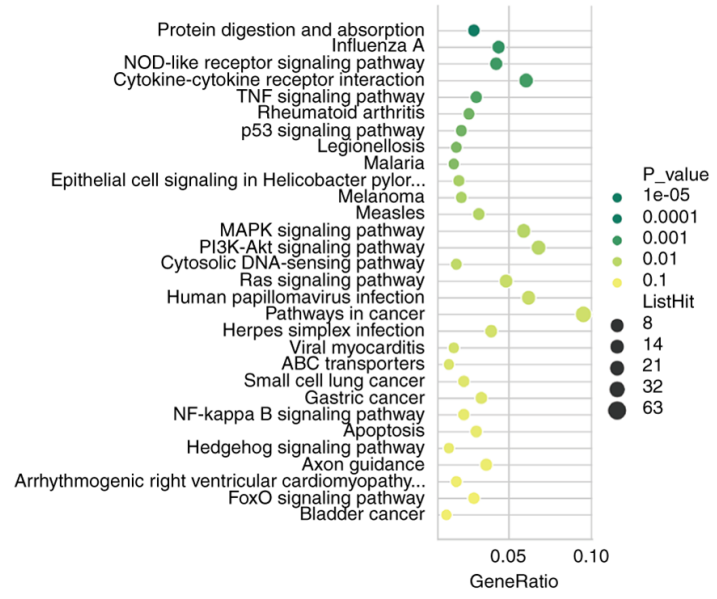

$\mathrm{H}$

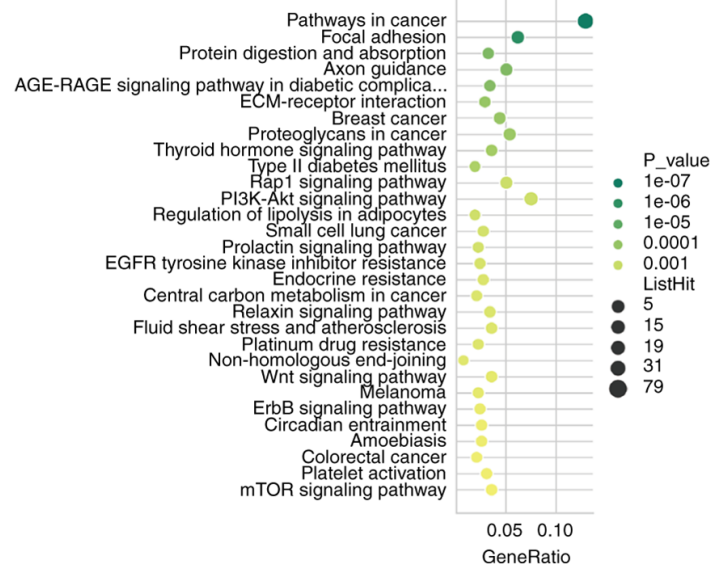

Figure 2. GO and pathway analysis of differentially expressed circRNAs and genes. (A) GO and (B) KEGG pathway analyses of upregulated circRNAs. (C) GO and (D) KEGG pathway analyses of downregulated circRNAs. (E) GO and (F) KEGG pathway analyses of upregulated genes. (G) GO and (H) KEGG pathway analyses of downregulated genes. GO, Gene Ontology; KEGG, Kyoto Encyclopedia of Genes and Genomes; circRNA, circular RNA. 


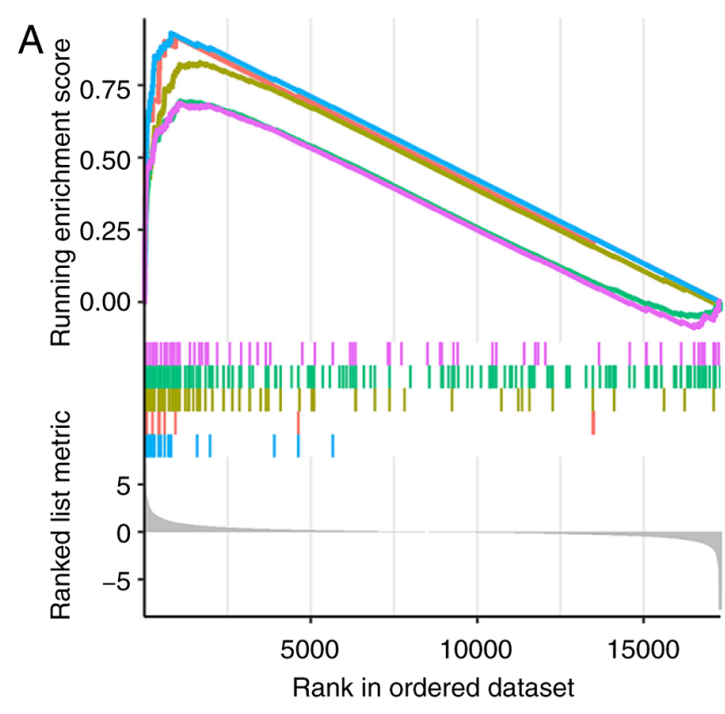

Response to extracellular matrix

Interferon alpha response

Interferon gamma response

IFNA response

TNF signaling up

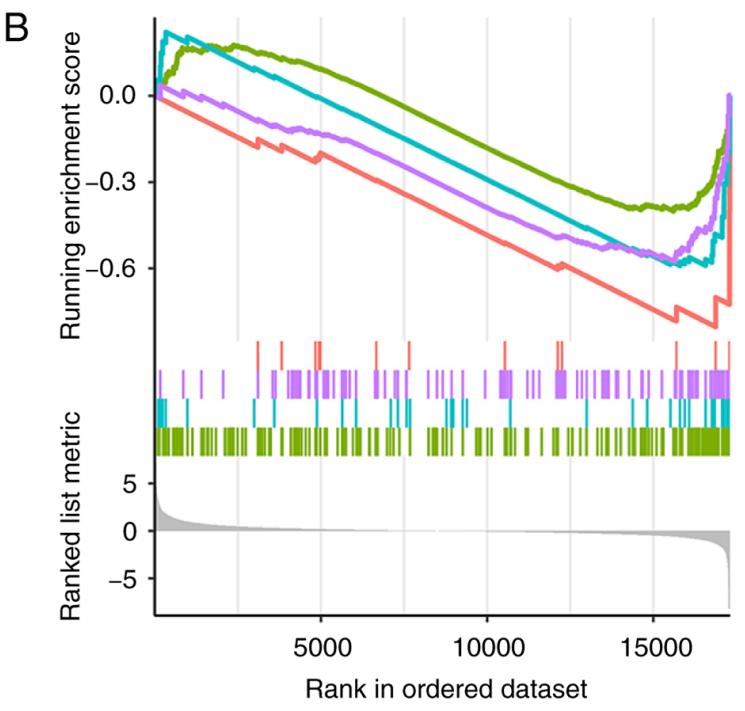

$$
\begin{gathered}
\text { IL3 pathway } \\
\text { Focal adhesion } \\
\text { Collagens }
\end{gathered}
$$

Signaling by PDGF

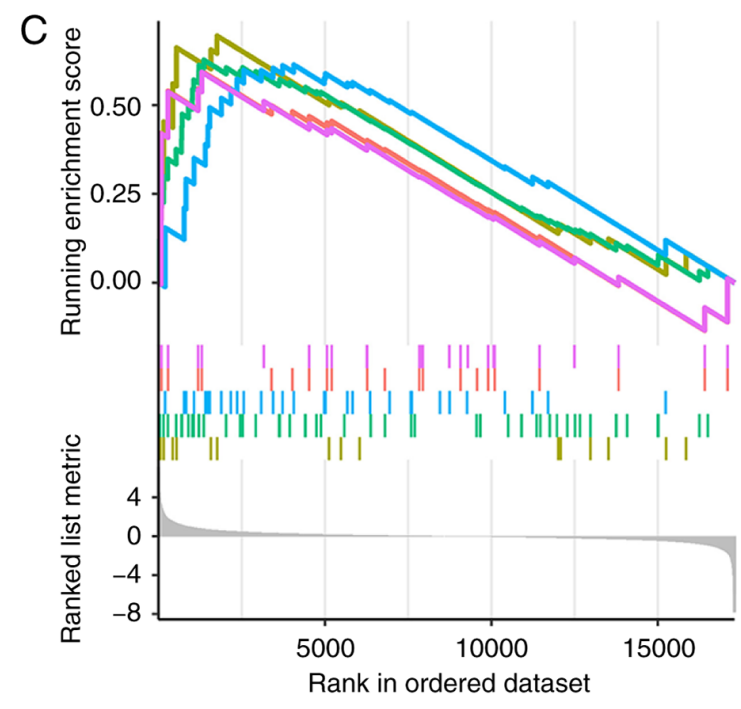

alpha-Linolenic acid metabolism

Ascorbate and aldarate metabolism

Cytosolic DNA-sensing pathway

Fructose and mannose metabolism

Linoleic acid metabolism

Figure 3. Gene Set Enrichment Analysis of differentially expressed genes. Significantly (A) upregulated and (B) downregulated signaling pathways in the lacrimal gland adenoid cystic carcinoma with high-grade transformation group. (C) Significantly upregulated pathways in cells.

by a poorer prognosis, higher local recurrence rate and propensity for cervical lymph node metastasis. In order to further study the molecular mechanism of LACC-HGT, DEMs in LACC and LACC-HGT paraffin-embedded tissues were identified. Meanwhile, the DECs and DEGs in LACC primary cell lines with and without HGT were also detected using a microarray. To the best of our knowledge, the current study is the first to comprehensively investigate the transcriptome circRNAs, miRNAs and genes profiles in LACC-HGT. ACC is an epithelial malignant tumor, which occurs mostly in the salivary glands and relatively rare in the lacrimal gland (33). The concept of 'HGT' was added to the salivary gland tumors of the World Health Organisation 'Classification of Head and Neck Tumors' in the 4th edition of 2017 (34). HGT can also occur in LACC (35). Therefore, it was hypothesized that miRNAs involved in SACC may also participate in LACC-HGT. Using combined bioinformatics analyses of GEO database, four candidate miRNAs associated with LACC-HGT were identified. In total, 16 DEMs were 

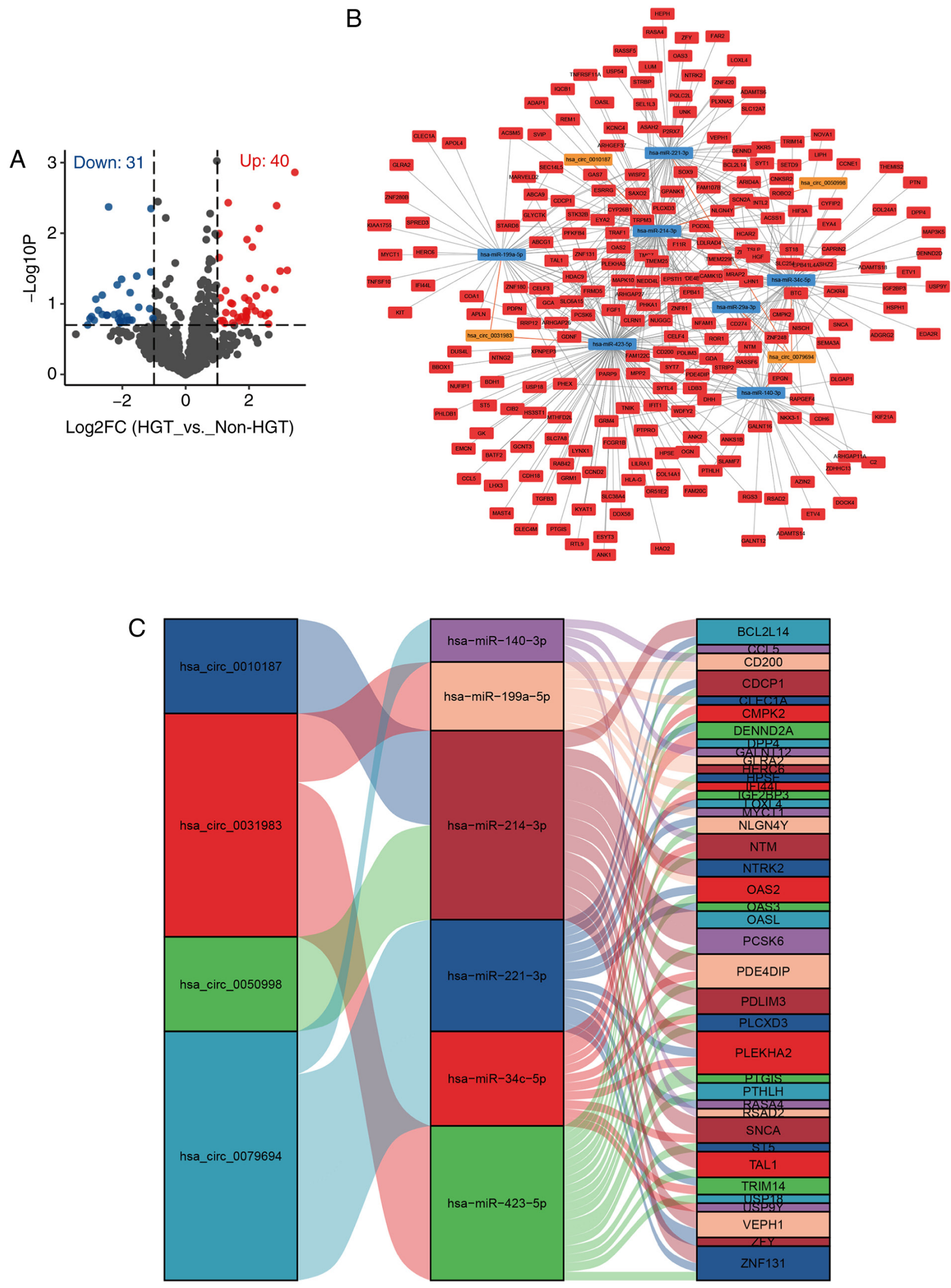

Figure 4. ceRNA regulatory network construction. (A) Volcano plot of differentially expressed miRNAs after adjustment of screening criteria. (B) Upregulated circRNA-mediated ceRNA regulatory network of lacrimal gland adenoid cystic carcinoma with high-grade transformation. Orange nodes are upregulated circRNAs, blue nodes are downregulated miRNAs that can be sponged by the corresponding circRNAs, and red nodes are upregulated genes that can be targeted by the corresponding miRNAs. Orange lines are circRNA-miRNA interactions, and gray lines are miRNA-mRNA interactions. (C) Sankey diagram of ceRNA regulatory network. circRNA, circular RNA; ce, competing endogenous; miR, microRNA. 


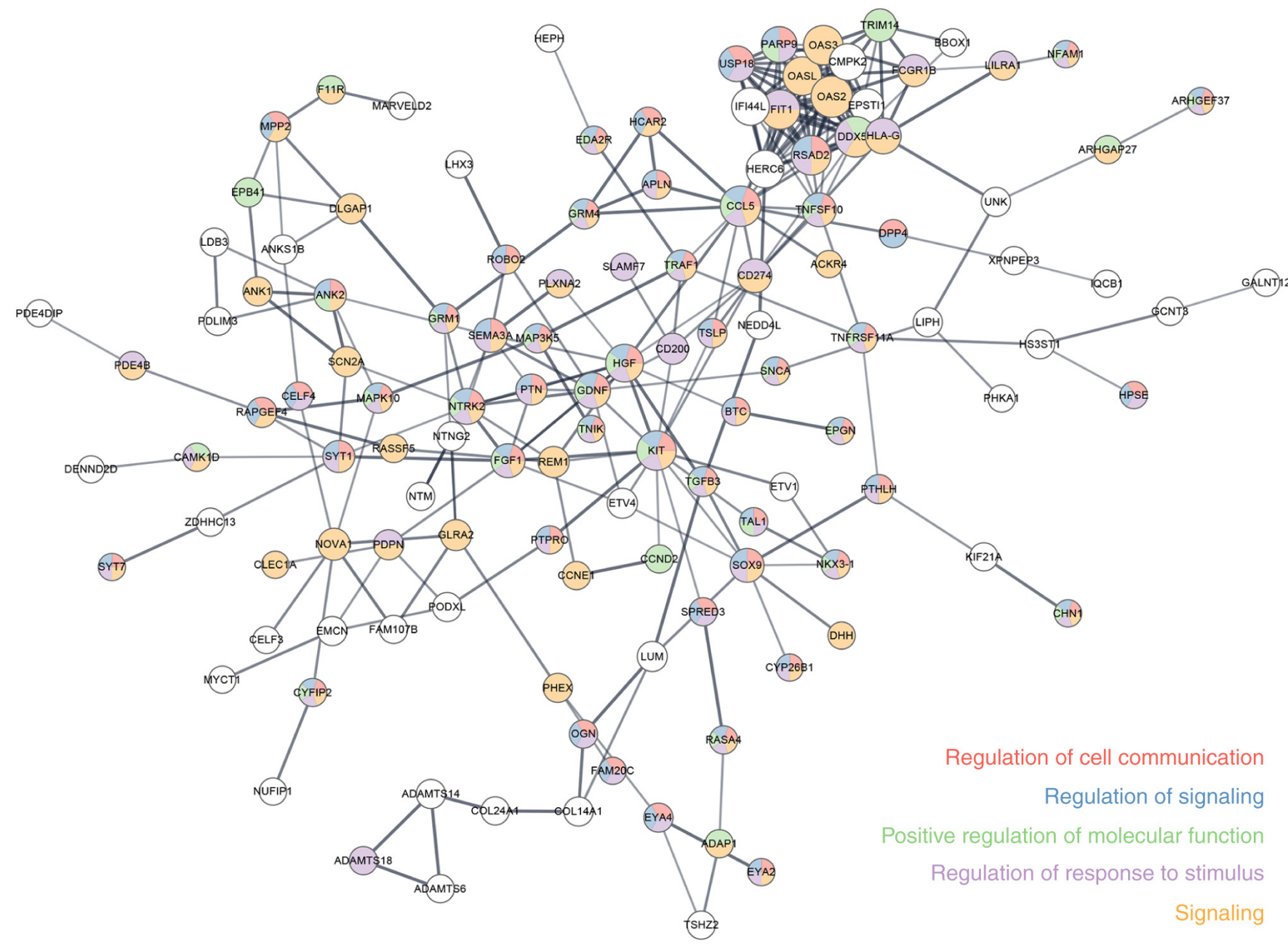

Figure 5. Protein-protein interaction network of proteins in competing endogenous RNA regulatory network.
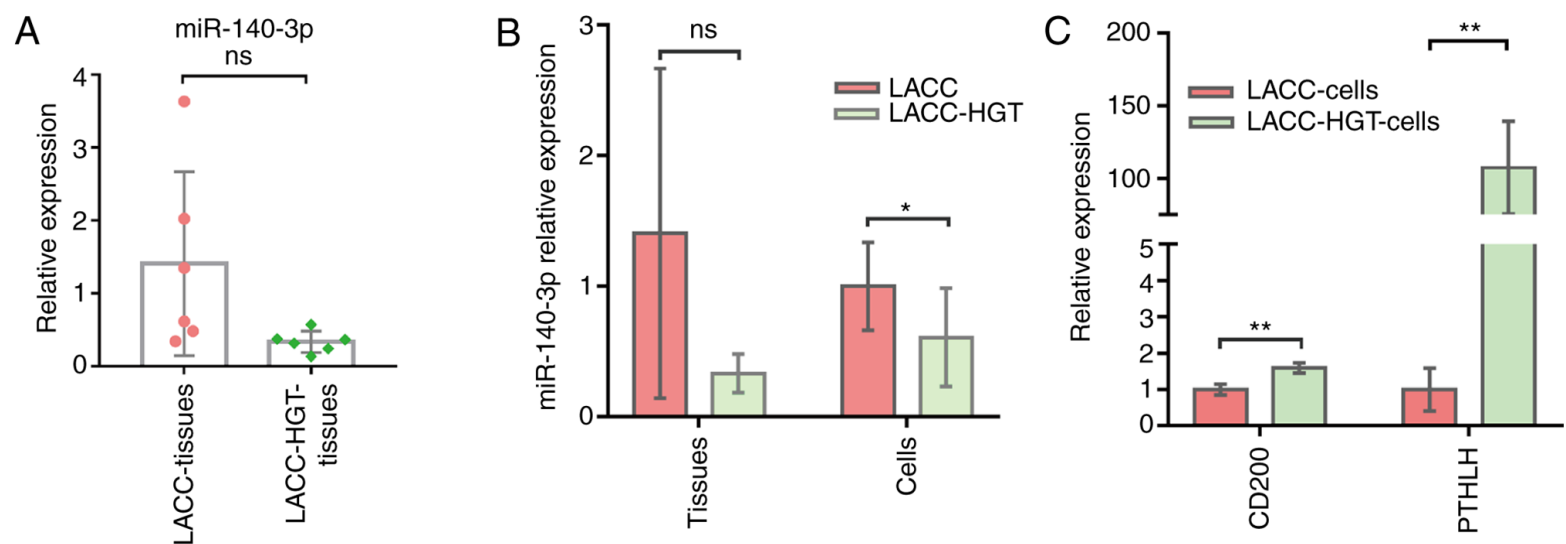

Figure 6. Reverse transcription-quantitative PCR results. (A) Downregulated expression of miR-140-3p in LACC-HGT tissues. (B) Expression of miR-140-3p in LACC and LACC-HGT tissues and primary cells. (C) CD200 and PTHLH were upregulated in LACC-HGT primary cells. ${ }^{*} \mathrm{P}<0.05$, ${ }^{* *} \mathrm{P}<0.01 \mathrm{vs}$. respective control. ns, not significant; LACC, lacrimal gland adenoid cystic carcinoma; HGT, high-grade transformation; miR, microRNA; PTHLH, parathyroid hormone-related protein.

detected. Previous studies on ACC mainly focused on the salivary gland, and miRNAs differentially expressed of SACC may also be involved in the malignant progression of LACC (36). Therefore, the overlapping results from the microarray and GEO database were analyzed, and four overlapping candidate miRNAs were identified; hsa-miR-140-3p, -140-5p, -574-3p and -636 Among them, hsa-miR-140-5p mediates inhibition of Smad2 and autophagy, inhibiting the survival and invasion potential of colorectal cancer stem cells (37). The 5' isomer miR with high expression of hsa-miR-140-3p contributes to the tumor-suppressive effects of miR-140 by reducing the proliferation and migration of breast cancer cells (38). However, there is 
no conclusive evidence that hsa-miR-574-3p and hsa-miR-636 are associated with tumors. The present study detected DEMs through miRNA microarrays, but further studies are needed to demonstrate the accuracy of the results and their biological role. For example, the expression of hsa-miR-140-3p and -140-5p in different tumors is not the same and the current data indicated that they were downregulated in LACC-HGT. Although candidate miRNAs were identified through the GEO database, this does not mean that other miRNAs do not play an important role in the occurrence and development of LACC-HGT.

Ultimately, differential expression of 653 circRNAs and 9,566 genes were detected. GO and KEGG pathway analyses of the DECs showed that they are mainly located in 'cytosol', 'membrane' and 'nucleoplasm', closely associated with 'translational initiation', 'nuclear export signal receptor activity', 'regulation of $\mathrm{G}_{2} / \mathrm{M}$ transition' and 'focal adhesion' and mainly involved in 'RNA transport', 'RNA degradation' and 'cell cycle' processes. DEGs were mainly located in 'plasma membrane', 'extracellular matrix', 'basement membrane and cytoplasm', closely associated with 'interferon signaling pathway', 'NF-кB signaling pathway', 'extracellular matrix' and 'cell proliferation' and mainly involved in 'protein digestion' and 'absorption'. DEG processes included 'cancer', 'p53 signaling pathway' and 'MAPK signaling pathway'. GSEA based on the DEGs indicated that several pathways may play important roles in LACC-HGT pathogenesis, mainly including the 'interferon $\alpha$ response pathway', 'IL-3 pathway' and some metabolic pathways. These signaling pathways are involved in the development of tumors, such as NF- $\mathrm{B}$, p53 and MAPK signaling pathways, which are canonical tumor-related signaling pathways (39-42). Interferon is a protein involved in a variety of functions, including antiviral and antimicrobial responses, apoptosis, control of the cell cycle, and the interferon signaling system plays an essential role in carcinogenic processes such a triple-negative breast cancer (43) and Kaposi's sarcoma (44).

Next, a circRNA-miRNA-mRNA ceRNA network was constructed using DECs, DEMs and DEGs. Ultimately, 77 ceRNA triplets were matched with the DEMs of LACC-HGT. In total, four circRNAs (hsa_circ_0079694, _0031983,_0050998 and _0010187), seven miRNAs (hsa-miR-140-3p, -199a-5p, -214-3p, -221-3p, -29a-3p, -34c-5p and $-423-5 p$ ) and 235 genes were involved in the ceRNA regulatory network. The function of these circRNAs is unclear. However, these miRNAs play an important role in tumorigenesis, among which miR-199a-5p is involved in the occurrence and development of a variety of tumors, such as colon and rectal cancer (45), uveal melanoma (46), oral cancer (47), glioma (48) and lung cancer (49). lncRNA XIST has anticancer effects on epithelial ovarian cancer cells through inverse downregulation of hsa-miR-214-3p (50). lncRNA LINC00968 inhibits cell proliferation and migration and angiogenesis in breast cancer through upregulation of prospero homeobox protein 1 by reducing hsa-miR-423-5p expression (51). The proteins in the ceRNA regulatory network were used to construct PPI regulation network, including 126 nodes and 261 edges. Candidate miRNAs (including miR-140-3p) also participated in the ceRNA regulatory network, so RT-qPCR was performed on miR-140-3p and its target genes in LACC-HGT tissues and primary cells. The results showed that the expression of miR-140-3p in
LACC-HGT tissues was downregulated and its target genes were upregulated. However, further exploration is needed to verify the specific underlying mechanisms.

In summary, the present analyses revealed that ceRNA regulation based on circRNA-miRNA-mRNA is a complex and sophisticated mechanism, and may have an essential regulatory role in the development of LACC-HGT. A ceRNA regulatory network in LACC-HGT was reported, with the comprehensive elucidation of the roles of ncRNAs in the pathogenesis of LACC-HGT. A limitation of this study is that even though DECs, DEMs and DEGs were detected by microarray, further investigation is still needed to validate the results and elucidate their roles and mechanism in LACC-HGT. Additional evidence should also be obtained to fully elucidate the identified ceRNA regulatory networks in LACC. Future biological studies may provide more detailed information to resolve the complex regulation of ceRNAs in LACC-HGT. Transwell, wound-healing assay and colony-formation assays may help identify the functions of DECs, DEMs and DEGs. Biological markers of LACC-HGT could be screened from a larger sample size. Fluorescence in situ hybridization would aid with quantitative analysis.

\section{Acknowledgements}

Not applicable.

\section{Funding}

The present study was supported by a grant from The National Natural Science Foundation of China (grant no. 81570872) and The Tianjin Key Clinical Discipline Construction Project (grant nos. TJLCZDXKT006 and TJLCZDXKQ024), we acknowledge the support received from Tianjin Medical University Eye Institute.

\section{Availability of data and materials}

The datasets used and/or analyzed during the present study are available from the corresponding author upon reasonable request. The datasets generated and/or analyzed during the present study are available in the GEO repository, [http://www.ncbi.nlm.nih.gov/geo].

\section{Authors' contributions}

MJ, XL, CZ, LZ, HDW, LD, TW, TL, YH performed the experiments. MJ, XL and TL confirmed the authenticity of all raw data. $\mathrm{MJ}, \mathrm{XL}$ and $\mathrm{CZ}$ conceived the project, drafted the initial manuscript and revised it for important intellectual content. TL and LZ collected the clinical data. HDW provided technical guidance. YH made the final diagnosis and performed surgeries for these patients. YH, LD and TL provided a critical review of the content. All authors have read and approved the final manuscript.

\section{Ethics approval and consent to participate}

The Institutional of Human Research Ethics Committee of the Tianjin Medical University Eye Hospital approved the 
study (Tianjin, China; approval no. 2017KY(L)-23), and all procedures were performed in accordance with the Declaration of Helsinki. All patients provided written informed consent.

\section{Patient consent for publication}

Not applicable.

\section{Competing interests}

The authors declare that they have no competing interests.

\section{References}

1. Ford JR, Rubin ML, Frank SJ, Ning J, Debnam JM, Bell D, El-Naggar A, Ferrarotto R and Esmaeli B: Prognostic factors for local recurrence and survival and impact of local treatments on survival in lacrimal gland carcinoma. Br J Ophthalmol: Jul 17, 2020 (Epub ahead of print). doi: 10.1136/bjophthalmol-2020-316142.

2. Sharma K, Rathi A, Khurana N, Mukherji A, Kumar V, Singh K and Bahadur AK: A retrospective study of 18 cases of adenoid cystic cancer at a tertiary care centre in Delhi. Indian J Cancer 47: 424-429, 2010.

3. Chen TY, Keeney MG, Chintakuntlawar AV, Knutson DL, Kloft-Nelson S, Greipp PT, Garrity JA, Salomao DR and Garcia JJ: Adenoid cystic carcinoma of the lacrimal gland is frequently characterized by MYB rearrangement. Eye (Lond) 31: 720-725, 2017.

4. Esmaeli B, Ahmadi M, Youssef A, Diba R, Amato M, Myers JN, Kies M and El-Naggar A: Outcomes in patients with adenoid cystic carcinoma of the lacrimal gland. Ophthalmic Plast Reconstr Surg 20: 22-26, 2004.

5. Wolkow N, Jakobiec F and Lee H: Long-term outcomes of globepreserving surgery with proton beam radiation for adenoid cystic carcinoma of lacrimal gland. Am J Ophthalmol 201: 84-85, 2019

6. Han J, Kim Y, Woo K and Sobti D: Long-term outcomes of eye-sparing surgery for adenoid cystic carcinoma of lacrimal gland. Ophthalmic Plast Reconstr Surg 34: 74-78, 2018.

7. Snyder M and Paulino A: Hybrid carcinoma of the salivary gland: Salivary duct adenocarcinoma adenoid cystic carcinoma. Histopathology 35: 380-383, 1999.

8. Nagao T: 'Dedifferentiation' and high-grade transformation in salivary gland carcinomas. Head Neck Pathol 7 (Suppl 1): S37-S47, 2013.

9. Kamio N, Tanaka Y, Mukai M, Ikeda E, Kuramochi S, Fujii M and Hosoda Y: A hybrid carcinoma: Adenoid cystic carcinoma and salivary duct carcinoma of the salivary gland. Virchows Archiv 430: 495-500, 1997.

10. Nagao T, Gaffey TA, Serizawa H, Sugano I, Ishida Y, Yamazaki K, Tokashiki R, Yoshida T, Minato H, Kay PA and Lewis JE: Dedifferentiated adenoid cystic carcinoma: A clinicopathologic study of 6 cases. Mod Pathol 16: 1265-1272, 2003.

11. White VA: Update on lacrimal gland neoplasms: Molecular pathology of interest. Saudi J Ophthalmol 26: 133-135, 2012.

12. Pusztaszeri $M$ and Brochu V: Metastatic adenoid cystic carcinoma with high-grade transformation ('dedifferentiation') in pleural effusion and neck lymph node: A diagnostic challenge on cytology? Diagn Cytopathol 48: 679-683, 2020.

13. Panni S, Lovering RC, Porras $\mathrm{P}$ and Orchard S: Non-coding RNA regulatory networks. Biochim Biophys Acta Gene Regul Mech 1863: 194417, 2020.

14. Zhang M, Zhang J, Zhang $\mathrm{H}$ and Tang $\mathrm{H}$ : miR-24-3p suppresses malignant behavior of lacrimal adenoid cystic carcinoma by targeting PRKCH to regulate p53/p21 pathway. PLoS One 11: e0158433, 2016.

15. Hao J, Jin X, Shi Y and Zhang H: miR-93-5p enhance lacrimal gland adenoid cystic carcinoma cell tumorigenesis by targeting BRMS1L. Cancer Cell Int 18: 72, 2018.

16. Salzman J: Circular RNA expression: Its potential regulation and function. Trends Genet 32: 309-316, 2016.

17. Salmena L, Poliseno L, Tay Y, Kats L and Pandolfi P: A ceRNA hypothesis: The Rosetta stone of a hidden RNA language? Cell 146: 353-358, 2011

18. Smillie CL, Sirey T and Ponting CP: Complexities of post-transcriptional regulation and the modeling of ceRNA crosstalk. Crit Rev Biochem Mol Biol 53: 231-245, 2018.
19. Lin T, Zhu L, Zhou B, Xie L, Lv J, Dong L and He Y: Establishment and characterization of a cell line from human adenoid cystic carcinoma of the lacrimal glands and a nude mouse transplantable model. Oncol Rep 33: 2797-2806, 2015.

20. Gao R, Cao C, Zhang M, Lopez MC, Yan Y, Chen Z, Mitani Y, Zhang L, Zajac-Kaye M, Liu B, et al: A unifying gene signature for adenoid cystic cancer identifies parallel MYB-dependent and MYB-independent therapeutic targets. Oncotarget 5: 12528-12542, 2014.

21. Kumar G, Kumar R, Pal MK, Pramanik N, Lahiri T, Gupta A and Pandey S: APT: An Automated Probe Tracker from gene expression data. IEEE/ACM Trans Comput Biol Bioinform: Dec 9, 2019 (Epub ahead of print). doi: 10.1109/TCBB.2019.2958345.

22. Kim B: How to reveal magnitude of gene signals: Hierarchical hypergeometric complementary cumulative distribution function. Evol Bioinform Online 14: 1176934318797352 , 2018.

23. Dudekula DB, Panda AC, Grammatikakis I, De S, Abdelmohsen K and Gorospe M: CircInteractome: A web tool for exploring circular RNAs and their interacting proteins and microRNAs. RNA Biol 13: 34-42, 2016.

24. Shannon P, Markiel A, Ozier O, Baliga NS, Wang JT, Ramage D, Amin N, Schwikowski B and Ideker T: Cytoscape: A software environment for integrated models of biomolecular interaction networks. Genome Res 13: 2498-2504, 2003.

25. Szklarczyk D, Gable AL,Lyon D, Junge A, Wyder S, Huerta-Cepas J, Simonovic M, Doncheva NT, Morris JH, Bork P, et al: STRING v11: Protein-protein association networks with increased coverage, supporting functional discovery in genome-wide experimental datasets. Nucleic Acids Res 47: D607-D613, 2019.

26. Schmittgen TD and Livak KJ: Analyzing real-time PCR data by the comparative C(T) method. Nat Protoc 3: 1101-1108, 2008.

27. Savithri V, Suresh R, Janardhanan M, Aravind T and Mohan M: Primary intraosseous adenoid cystic carcinoma with widespread skeletal metastases showing features of high-grade transformation. Head Neck Pathol: Sep 21, 2020 (Epub ahead of print). doi: 10.1007/s12105-020-01228-x.

28. Falzone L, Grimaldi M, Celentano E, Augustin L and Libra M: Identification of modulated MicroRNAs associated with breast cancer, diet, and physical activity. Cancers 12: 2555, 2020.

29. Filetti V, Falzone L, Rapisarda V, Caltabiano R, Eleonora Graziano AC, Ledda C and Loreto C: Modulation of microRNA expression levels after naturally occurring asbestiform fibers exposure as a diagnostic biomarker of mesothelial neoplastic transformation. Ecotoxicol Environ Saf 198: 110640, 2020.

30. Liu Y, Di G, Hu S, Zhao T, Xu X, Wang X and Chen P: Expression profiles of CircRNA and mRNA in lacrimal glands of AQP5 mice with primary dry eye. Front Physiol 11: 1010, 2020.

31. Han D, Li J, Wang H, Su X, Hou J, Gu Y, Qian C, Lin Y, Liu X, Huang M, et al: Circular RNA circMTO1 acts as the sponge of microRNA-9 to suppress hepatocellular carcinoma progression. Hepatology 66: 1151-1164, 2017.

32. Hansen TB, Jensen TI, Clausen BH, Bramsen JB, Finsen B, Damgaard CK and Kjems J: Natural RNA circles function as efficient microRNA sponges. Nature 495: 384-388, 2013.

33. Nakaguro M, Faquin WC, Baloch ZW, Cantley RL, Compton ML, Ely KA, Holmes BJ, Hu R, Kerr DA, Montone KT, et al: Fine needle aspiration of salivary gland carcinomas with high-grade transformation: A multi-institutional study of 22 cases and review of the literature. Cancer Cytopathol: Nov 19, 2020 (Epub ahead of print). doi: $10.1002 /$ cncy. 22388 .

34. Seethala R and Stenman G: Update from the 4th Edition of the World Health Organization Classification of Head and Neck Tumours: Tumors of the salivary gland. Head Neck Pathol 11: 55-67, 2017.

35. Seethala R, Hunt J, Baloch Z, Livolsi V and Leon Barnes E: Adenoid cystic carcinoma with high-grade transformation: A report of 11 cases and a review of the literature. Am J Surg Pathol 31: 1683-1694, 2007.

36. Kiss O, Tőkés A, Spisák S, Szilágyi A, Lippai N, Székely B, Szász AM and Kulka J: Breast- and salivary gland-derived adenoid cystic carcinomas: potential post-transcriptional divergencies. A pilot study based on miRNA expression profiling of four cases and review of the potential relevance of the findings. Pathol Oncol Res 21: 29-44, 2015.

37. Zhai H, Fesler A, Ba Y, Wu S and Ju J: Inhibition of colorectal cancer stem cell survival and invasive potential by hsa-miR-140-5p mediated suppression of Smad2 and autophagy. Oncotarget 6: 19735-19746, 2015. 
38. Salem O, Erdem N, Jung J, Münstermann E, Wörner A Wilhelm H, Wiemann S and Körner C: The highly expressed 5 'isomiR of hsa-miR-140-3p contributes to the tumor-suppressive effects of miR-140 by reducing breast cancer proliferation and migration. BMC Genomics 17: 566, 2016.

39. Joerger A and Fersht A: The p53 pathway: Origins, inactivation in cancer, and emerging therapeutic approaches. Ann Rev Biochem 85: 375-404, 2016.

40. Sanchez-Vega F, Mina M, Armenia J, Chatila WK, Luna A La KC, Dimitriadoy S, Liu DL, Kantheti HS, Saghafinia S, et al: Oncogenic signaling pathways in the cancer genome atlas. Cell 173: 321-337.e10, 2018.

41. Tang $\mathrm{C}$ and Zhu G: Classic and novel signaling pathways involved in cancer: Targeting the NF- $\mathrm{BB}$ and Syk signaling pathways. Curr Stem Cell Res Ther 14: 219-225, 2019.

42. Guo Y, Pan W, Liu S, Shen Z, Xu Y and Hu L: ERK/MAPK signalling pathway and tumorigenesis. Exp Ther Med 19: 1997-2007, 2020.

43. Park IA, Hwang SH, Song IH, Heo SH, Kim YA, Bang WS, Park HS, Lee M, Gong G and Lee HJ: Expression of the MHC class II in triple-negative breast cancer is associated with tumor-infiltrating lymphocytes and interferon signaling. PLoS One 12: e0182786, 2017.

44. Joo CH, Shin YC, Gack M, Wu L, Levy D and Jung JU: Inhibition of interferon regulatory factor 7 (IRF7)-mediated interferon signal transduction by the Kaposi's sarcoma-associated herpesvirus viral IRF homolog vIRF3. J Virol 81: 8282-8292, 2007.

45. Slattery $M$, Lundgreen A, Bondurant $K$ and Wolff $R$ : Interferon-signaling pathway: Associations with colon and rectal cancer risk and subsequent survival. Carcinogenesis 32 1660-1667, 2011.
46. Falzone L, Romano GL, Salemi R, Bucolo C, Tomasello B, Lupo G, Anfuso CD, Spandidos DA, Libra M and Candido S: Prognostic significance of deregulated microRNAs in uveal melanomas. Mol Med Rep 19: 2599-2610, 2019.

47. Wei D, Wang W, Shen B, Zhou Y, Yang X, Lu G, Yang J and Shao Y: MicroRNA-199a-5p suppresses migration and invasion in oral squamous cell carcinoma through inhibiting the EMT-related transcription factor SOX4. Int J Mol Med 44: 185-195, 2019.

48. Zhang C, Chen Q, Zhu J and Liu Z: MicroRNA-199a-5p regulates glioma progression via targeting MARCH8. Eur Rev Med Pharmacol Sci 23: 7482-7487, 2019.

49. Ahmadi A, Khansarinejad B, Hosseinkhani S, Ghanei M and Mowla S: miR-199a-5p and miR-495 target GRP78 within UPR pathway of lung cancer. Gene 620: 15-22, 2017.

50. Wang C, Qi S, Xie C, Li C, Wang P and Liu D: Upregulation of long non-coding RNA XIST has anticancer effects on epithelial ovarian cancer cells through inverse downregulation of hsa-miR-214-3p. J Gynecol Oncol 29: e99, 2018.

51. Sun X, Huang T, Zhang C, Zhang S, Wang Y, Zhang Q and Liu Z: Long non-coding RNA LINC00968 reduces cell proliferation and migration and angiogenesis in breast cancer through up-regulation of PROX1 by reducing hsa-miR-423-5p. Cell Cycle 18: 1908-1924, 2019.

(c) (1) $(9)$ This work is licensed under a Creative Commons Attribution-NonCommercial-NoDerivatives 4.0 International (CC BY-NC-ND 4.0) License. 